\title{
On the origin of POU5F1
}

Stephen Frankenberg ${ }^{*}$ and Marilyn B Renfree

\begin{abstract}
Background: Pluripotency is a fundamental property of early mammalian development but it is currently unclear to what extent its cellular mechanisms are conserved in vertebrates or metazoans. POU5F1 and POU2 are the two principle members constituting the class $\vee$ POU domain family of transcription factors, thought to have a conserved role in the regulation of pluripotency in vertebrates as well as germ cell maintenance and neural patterning. They have undergone a complex pattern of evolution which is poorly understood and controversial.

Results: By analyzing the sequences of POU5F1, POU2 and their flanking genes, we provide strong indirect evidence that POU5F1 originated at least as early as a common ancestor of gnathostomes but became extinct in a common ancestor of teleost fishes, while both POU5F1 and POU2 survived in the sarcopterygian lineage leading to tetrapods. Less divergent forms of POU5F1 and POU2 appear to have persisted among cartilaginous fishes.

Conclusions: Our study resolves the controversial evolutionary relationship between teleost pou2 and tetrapod POU2 and POU5F1, and shows that class V POU transcription factors have existed at least since the common ancestor of gnathostome vertebrates. It provides a framework for elucidating the basis for the lineage-specific extinctions of POU2 and POU5F1.
\end{abstract}

\section{Background}

Loss of potency during differentiation is fundamental to the development of complex metazoans. Pluripotent embryonic cells are able to give rise ultimately to all tissues of the adult body. In at least some mammals, pluripotency can be "captured" in vitro in the form of indefinitely selfrenewing embryonic stem (ES) cells. Thus ES cells can serve as a model for the differentiation of their in vivo counterparts into ectoderm, mesoderm and endoderm derivatives.

POU5F1 (also called OCT4 or OCT3/4) is a central regulator of pluripotency in mammals. In the mouse, deletion of Pou $5 \mathrm{fl}$ causes loss of pluripotency in the inner cell mass and differentiation to trophoblast, revealing its earliest developmental role [1]. POU5F1 is also a potent reprogramming factor capable of facilitating the derivation of induced pluripotent stem (iPS) cells [2,3]. Conditional knockout of Pou $5 f 1$ in mouse primordial germ cells results in their apoptosis [4], showing that the role of POU5F1 is not exclusively restricted to preventing differentiation.

POU2 is a vertebrate paralog of POU5F1 that has been best characterized in zebrafish. Curiously, some vertebrate

\footnotetext{
* Correspondence: srfr@yahoo.co.uk

Department of Zoology, University of Melbourne, Melbourne, VIC 3010,

Australia
}

lineages, such as salamanders, marsupials and monotremes, have preserved both POU2 and POU5F1 in their genomes while in other vertebrates one or the other gene has become extinct [5-7]. Thus squamate reptiles and eutherian mammals have only POU5F1 while birds and frogs have only POU2 (called POUV in birds). In Xenopus, POU2 is present as three tandem copies - OCT25, OCT60 and OCT 90.

For reasons that are not fully clear, teleost pou 2 was recently renamed pou $5 f 1$ despite multiple pieces of evidence for a closer affinity to POU2 orthologs of tetrapods. Onichtchouk [8] argued that since orthologous genes are defined "as originating from a single ancestral gene in the last common ancestor of the compared genomes", teleost pou 2 is orthologous to mammalian POU5F1. However, by the same argument, teleost pou 2 is also orthologous to tetrapod POU2 orthologs, thus obviating the need for a name change. Teleost pou 2 shares more sequence similarity as well as conserved synteny with tetrapod POU2 [5,6], but perhaps more importantly, it was not proven whether the duplication event giving rise to each paralog occurred after or before the common ancestor of tetrapods and teleost fishes. If the latter, POU5F1 must have become extinct in teleosts as it has in some other tetrapod lineages such as birds and frogs. 
POU5F1 and POU2 share a five-exon genomic structure that is characteristic of the class V POU family. Exons 1 and 5 encode the poorly conserved $\mathrm{N}$ - and C-terminal transactivation domains, respectively, while Exons 2 to 4 encode the highly conserved POU-domain, which comprises the POUspecific domain and the POU-homeodomain separated by a short linker region [9-11].

\section{Results \\ Newly identified POU2 and POU5F1 orthologs in vertebrates}

To gain insight into the origins of the class V POU family of transcription factors in vertebrates, BLAST searches were performed for sequences homologous to mammalian POU2 and POU5F1. Previously unreported orthologs of POU5F1 were identified from a large number of vertebrate species, including the painted turtle (Chrysemys picta bellii), Indian python (Python molurus) and coelacanth (Latimeria chalumnae). POU2 orthologs were also identified in many species, including the alligator (Alligator mississippiensis), painted turtle, coelacanth and spotted gar (Lepisosteus oculatus).

The avian POU2 ortholog - POUV - was identified in genome assemblies of the turkey (Meleagris gallopavo), medium ground finch (Geospiza fortis) and budgerigar (Melopsittacus undulatus), adding to the previously identified orthologs from chicken [12] and zebra finch [5]. Conserved open reading frames orthologous to chicken Exon 1 could not be identified in other avian species. As chicken Exon 1 was previously identified as unlikely to be homologous to Exon 1 from non-avian orthologs [5], all available avian genomes were re-examined. Low stringency BLAST searches identified a single sequence (Ti 224571611) from the chicken whole genome shotgun (WGS) trace archives with homology to the proximal promoter and 5' part of Exon 1 of non-avian POU2 orthologs (see below). In addition, a primordial germ cell-derived partial chicken EST (GenBank accession DR410403) included sequence with clear homology to the 3' part of Exon 1 from non-avian POU2 orthologs. The apparent absence of both the proximal POU2 promoter and the "canonical" Exon 1 in other birds is probably due to gaps in their respective genome assemblies, suggesting that features of this region impart recalcitrance to sequencing. We conclude that the previously published cDNA for chicken POUV represents a rare or non-canonical chickenspecific transcript (retaining the first intron) that was selectively isolated due to the PCR-based methods used.

Alignment of a broad selection of POU2 and POU5F1 translated sequences (Additional file 1) showed almost no conservation within the $\mathrm{N}$-terminal region between paralogs. However, a short motif with the consensus sequence (K/R)XWYXF was moderately well conserved in both POU2 and POU5F1 (Figure 1), the first time a sequence signature conserved in the $\mathrm{N}$-terminal domain of all family members has been identified. The previously noted N-terminal sequence MAGH and the deletion of a single arginine residue within the POU-homeodomain [5], as well as an aspartic acid instead of glutamic acid at a site within the POU-specific domain identified by $\mathrm{Ye}$ and colleagues [13], were among the few fully conserved signatures specifically characterizing POU5F1 orthologs. We also noted that a second single-residue deletion in the linker region separating the POU-specific and POUhomeodomains of POU5F1 is specific to Boreoeutheria, as it was not present in the elephant (Afrotheria), armadillo (Xenarthra) or any other vertebrate (Figure 1), suggesting a modification in function of POU5F1 among some eutherians.

\section{A multigenic duplication gave rise to POU2 and POU5F1}

To gain further insight into the evolution of POU2 and POU5F1 and to confirm orthology where possible, we examined their synteny with other genes in available vertebrate genome assemblies (summarized in Figure 2). As previously reported [5,6], POU2 is flanked by orthologs of NPDC1 and FUT7 in all vertebrate genomes for which synteny could be determined. In the coelacanth and turtle genomes, POU5F1 is flanked by a previously unreported paralog of NPDC1 - a 9-exon gene expressed in differentiating neuronal cells $[14,15]$ - which we call NPDC1L (NPDC1-like), indicating that the original duplication event that generated POU5F1 and POU2 was of a multigenic region. A search for NPDC1/NPDC1L homologs in other vertebrates identified sequences in several squamate reptiles, including python, anole lizard (Anolis carolinensis) and Schlegel's Japanese gecko (Gekko japonicus). Phylogenetic analysis showed that these sequences represent NPDC1L and not NPDC1 (Figure 3 and Additional file 2). Thus extinction of POU2 in squamate reptiles [5] was apparently associated with deletion of a larger, multigenic region that also included NPDC1. An additional rearrangement in the mammalian lineage resulted in $D D X 39 B$ lying upstream of POU5F1 and the possibly simultaneous extinction of NPDC1L. In the common ancestor of therian mammals (after their split with monotremes), the $\mathrm{H} 2$ major histocompatibility complex was inserted between $D D X 39 B$ and POU5F1 (Figure 2).

\section{The duplication that gave rise to POU5F1 occurred in a common ancestor of gnathostomes}

The apparent absence of POU5F1 in all non-sarcopterygian (for example, teleost fish) genomes at first glance suggested that the origin of POU5F1 by duplication of POU2 is specific to the sarcopterygian lineage, or at least cannot be proven otherwise. However, the demonstration (above) that the duplication included at least one flanking gene, NPDC1/ $N P D C 1 L$, provided an alternative strategy for determining 


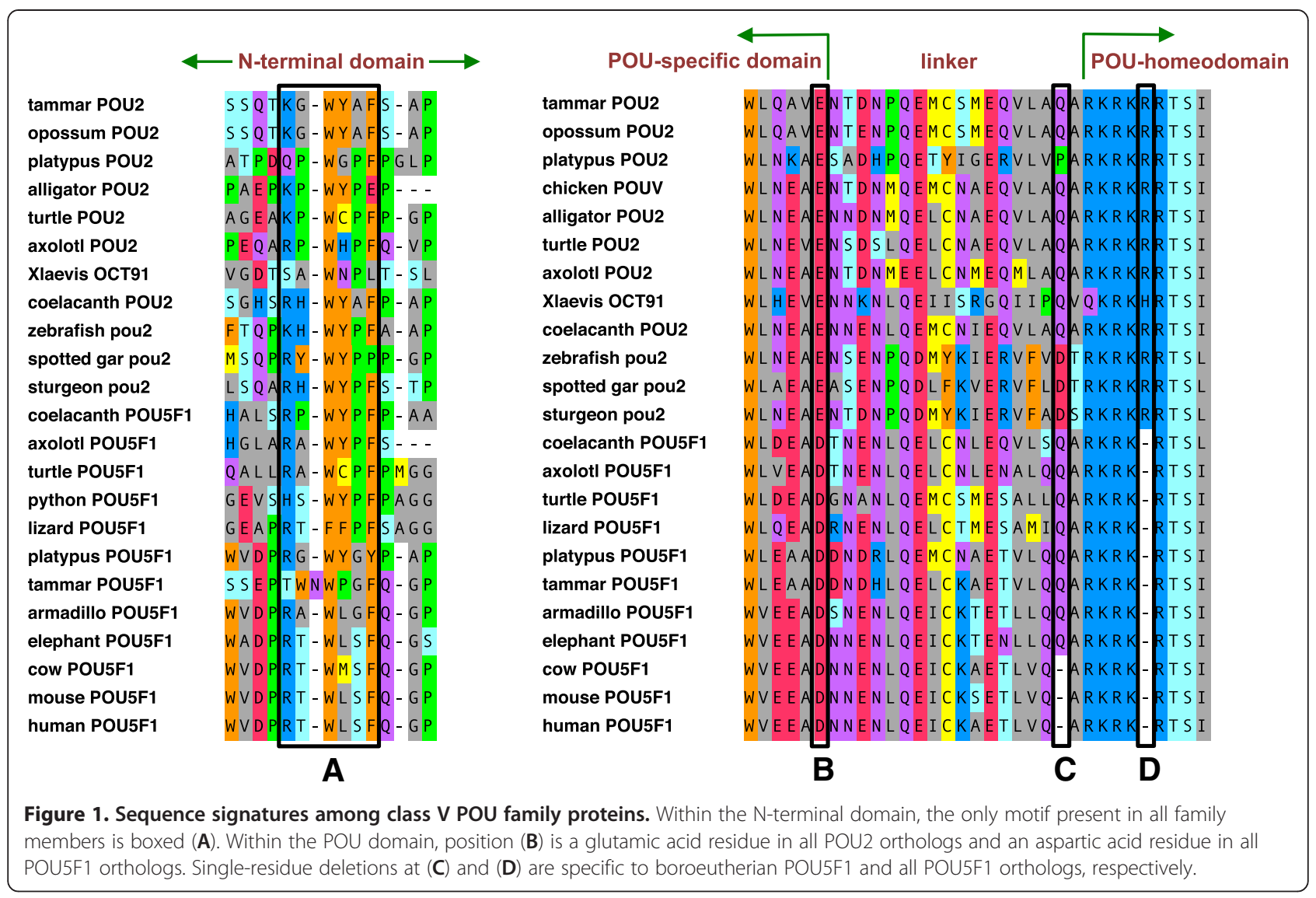

its timing. We, therefore, searched for homologs of NPDC1 and NPDC1L in cartilaginous fishes (class Chondrichthyes), focusing on Exons 5 to 9 as these are the best conserved. The identified sequences are summarized in Figure 4. Three whole genome shotgun (WGS) contigs were identified from the elephantfish (Callorhinchus callorynchus, subclass Holocephali), which included Exons 5 to 6,7 to 8 and 9, respectively. These presumably form part of a common gene but this was not assumed for the purpose of this analysis. Six WGS contigs were identified from the little skate (Leucoraja erinacea, subclass Elasmobranchii), each containing a single exon. These included two homologs of Exon 5, one of Exon 6, two of Exon 8 and one of Exon 9. Thus the little skate genome appears to contain at least two homologs of NPDC1/NPDC1L. Lastly, multiple overlapping expressed sequence tags (ESTs) from the spiny dogfish (Squalus acanthias, subclass Elasmobranchii) were identified, which together spanned almost the full coding region. These were combined in silico to produce a single sequence for analysis.

To maximize statistical power, we first compared the translated dogfish sequence (the only chondrichthyan sequence spanning Exons 5 to 8) with NPDC1 and NPDC1L orthologs of other species, including a tunicate (Ciona savignyi) NPDC1/NPDC1L homolog as an outgroup. The dogfish sequence clustered with NPDC1L orthologs with a significant bootstrap value using three different methods for generating consensus phylogenetic trees (maximum parsimony, maximum likelihood and neighbor-joining) (Figure 3A). In a comparison with the sequences from coelacanth, a species with both NPDC1L and NPDC1 (to control for lineage-specific differences in divergence rate), the dogfish sequence was clearly more similar to NPDC1L than to NPDC1, indicating that its clustering with NPDC1L in the consensus trees was not simply due to more rapid divergence from an ancestral NPDC1-like sequence (Figure $3 \mathrm{E}$ ). This indicated that a gene more similar to NPDC1L than to NPDC1 has existed since at least as early as the common ancestor of Chondrichthyes and Osteichthyes, and that duplication of an NPDC1/ $N P D C 1 L$ ancestral gene must have occurred before the split between Sarcopterygii and Actinopterygii, since both groups have NPDC1 orthologs that are more similar to each other than to NPDC1L. To examine whether the duplication occurred even earlier in a common ancestor of Chondrichthyes and Osteichthyes, we performed phylogenetic analyses of the other chondrichthyan sequences from elephantfish and little skate (Figure 3B-D). Both of the elephantfish sequences (spanning Exons 5 to 6 and 7 to 8 , respectively) clustered with NPCD1 orthologs and 


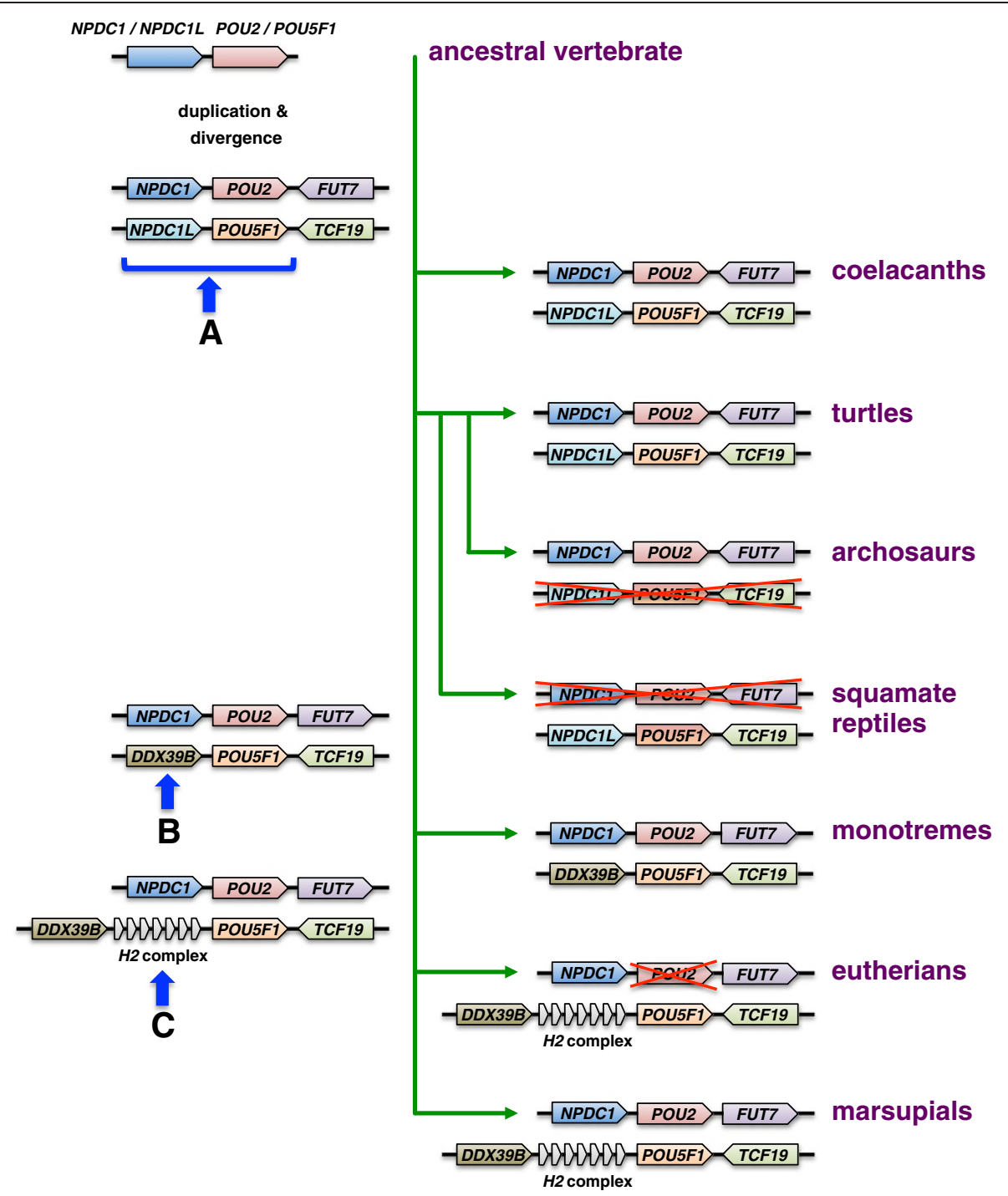

Figure 2. Synteny at the POU5F1 and POU2 loci in sarcopterygians. The synteny of POU5F1 and POU2 with other genes in extant species is shown on the right-hand side. Proposed ancestral genomic rearrangements that explain the current synteny are shown on the left-hand side. (A) A multigenic duplication in a sarcopterygian ancestor gave rise to POU5F1 and NPDC1L, causing POU5F1 to flank TCF19 and POU2 to flank FUT7. (B) In a common ancestor of mammals, deletion of NPDC1L caused POU5F1 to flank DDX39B. The orientation of FUT7 also was inverted. (C) In a common ancestor of therian mammals, the H2 major histocompatibility complex became inserted between POU5F1 and DDX39B.

were separate from the dogfish sequence and NPDC1L orthologs, regardless of the exon analyzed or the method used. For the little skate, one of the two Exon 5 sequences and one of the two Exon 8 sequences clustered with the dogfish sequence regardless of the analysis method and with significant bootstrap values for three of the six analyses (one for Exon 5 and two for Exon 8), indicating that these sequences are orthologous to the dogfish sequence. The other little skate Exon 5 and Exon 8 sequences, plus the Exon 6 sequence, each clustered with an elephantfish sequence to the exclusion of all other sequences in almost every case (8/9), with only one (non-significant) exception (Exon 5 - maximum parsimony; Figure 3B). Bootstrap values for this clustering were significant in three of the other eight analyses (Exon 6 - maximum parsimony; Exon 8 - maximum likelihood and neighbor-joining). These results strongly suggested that chondrichthyans collectively have both NPDC1 and NPDC1L paralogs and that both are present in the little skate genome. To exclude the possibility that the putative NPDC1 ortholog (in elephantfish and little skate) is a chondrichthyan-specific paralog of the dogfish sequence, we compared the two elephantfish sequences (Exons 5 to 6 and 7 to 8) to coelacanth NPDC1 and NPDC1L (Figure 3E). Both elephantfish sequences were more similar to coelacanth NPDC1 than to either the dogfish sequence or coelacanth NPDC1L, strongly arguing against a scenario in which the elephantfish sequences are derived from a chondrichthyan-specific 


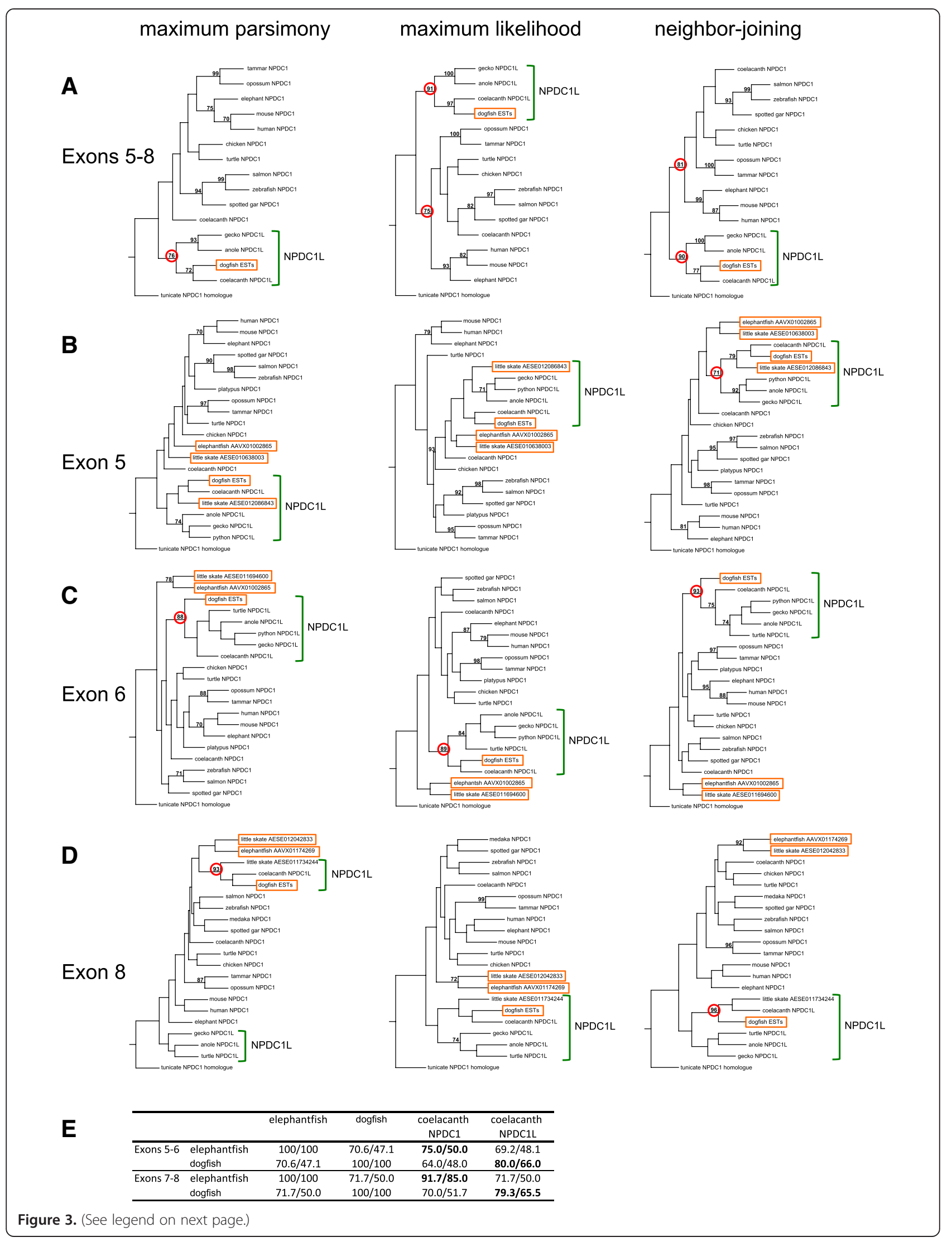


(See figure on previous page.)

Figure 3. Phylogenetic analysis of NPDC1/NPDC1L homologs in gnathostomes. (A-D) Phenograms of phylogenetic analyses alignments of translated sequences of NPDC1, NPDC1L and chondrichthyan sequences analyzed by maximum parsimony, maximum likelihood and neighborjoining methods, using a tunicate NPDC1/NPDC1L homolog as an outgroup. Alignments are presented in Additional file 2. Only significant bootstrap values $(>70 \%)$ are shown and those relevant to the text are circled in red. Chondrichthyan sequences are boxed. NPDC1L sequences, including putative chondrichthyan orthologs, are bracketed. (E) Percentage of similarity/identity values comparing translated elephantfish and dogfish sequences to each other and to coelacanth NPDC1 and NPDC1L. The percentages highlighted in bold show that the elephantfish sequences are most similar to coelacanth NPDC1 while the dogfish sequence is most similar to coelacanth NPDC1L, indicating respective orthology when combined with the phenogram data.

duplication of an ancestral NPDC1/NPDC1L precursor that was more similar to extant NPDC1L orthologs than to NPDC1 orthologs. It may thus be concluded that orthologs of both NPDC1 and NPDC1L are present among cartilaginous fishes and, therefore, that the duplication event giving rise to POU2 and POU5F1 must have occurred at least as early as a common ancestor of extant gnathostomes.

\section{Putative POU2 and POU5F1 orthologs are present in chondrichthyans}

Since the duplication that gave rise to NPDC1 and $N P D C 1 L$ can be reasonably assumed to have occurred in a common ancestor of cartilaginous fishes and other jawed vertebrates, we searched chondrichthyan databases thoroughly for homologs of POU2 and POU5F1. The identified sequences are summarized in Figure 4. In the elephantfish, we identified a single WGS contig encoding Exons 2 and 3 and a separate contig encoding Exon 5. In the little skate, we identified a partial sequence for Exon 1 and two homologs of each of Exons 3, 4 and 5, all on separate contigs. Thus, while it was unclear which of the identified exons collectively form part of a common gene, at least two genes encoding class V POU domain transcription factors exist in the little skate genome. Although synteny with other genes could not be determined from either of the chondrichthyan genome assemblies, various sequence signatures mostly resembled POU2 rather than POU5F1, including a lack of the single arginine deletion within the POU-specific domain of POU5F1 (Figure 1) [5]. This could be explained by a lineage-specific duplication of POU2 in the little skate, similar to the tandem POU2 triplication found in Xenopus. However, the presence of a single homolog of both NPDC1/NPDC1L and POU2/POU5F1 in the elephantfish but two homologs of each in the little skate suggested the presence of both POU 2 and POU5F1 orthologs in the latter species. To test this, we performed phylogenetic analysis of translated sequences for each exon (Figure 5 and Additional file 3). For the elephantfish sequences, Exons 2, 3 and 5 generally clustered with POU2 orthologs and this was highly significant for one analysis of Exon 5 (maximum likelihood; bootstrap value $90 \%$ ). This suggested that the elephantfish contains a single ortholog of POU2. The elephantfish sequences always clustered with one little skate sequence to the exclusion of all others (significant bootstrap value for all three analyses of each exon), indicating orthology. The remaining little skate sequences (Exon1, Exon 3, Exon $4(\times 2)$ and Exon 5) generally clustered non-significantly with either POU2 or POU5F1 orthologs, with two notable exceptions. The little skate Exon 1 sequence clustered with POU5F1 orthologs for all three methods of analysis. For one analysis this was highly significant (maximum parsimony - bootstrap 94\%). One of the little skate Exon 5 sequences clustered non-significantly with POU2 orthologs for one analysis method (maximum parsimony) but clustered highly significantly with POU5F1 orthologs for the other two methods (maximum likelihood - 90\%; neighbor-joining - 92\%).

Combined, the above data suggest that orthologs of both POU2 and POU5F1 exist among cartilaginous fishes. Although the identity of every WGS contig cannot be assigned with confidence, evidence suggests that the little skate has both a POU2 and a POU5F1 ortholog, while the elephantfish has only a POU2 ortholog. This is consistent with the presence of both NPDC1 and NPDC1L orthologs in the little skate, but only an NPDC1L ortholog in the elephantfish.

\section{Discussion}

Our data show that the duplication that gave rise to POU5F1 and POU2 occurred in a common gnathostomal ancestor. This can be deduced by combining two crucial pieces of evidence. First, conserved synteny shows that the duplication was multigenic and also gave rise to the paralogs NPDC1 and NPDC1L. Second, orthologs of both NPDC1 and NPDC1L were identified in cartilaginous fishes. Consistent with this deduction, we identified sequences in cartilaginous fishes that appear to correspond to either POU2 or POU5F1. Orthologs of both POU2 and POU5F1 are likely to still be present in the genome of the little skate, although their sequences appear less divergent from each other than they are in higher vertebrates. We also predict that an ortholog of POU5F1 is present in the spiny dogfish, since this species also retains an ortholog of NPDC1L, but POU5F1 is presumably extinct in the elephantfish lineage. 


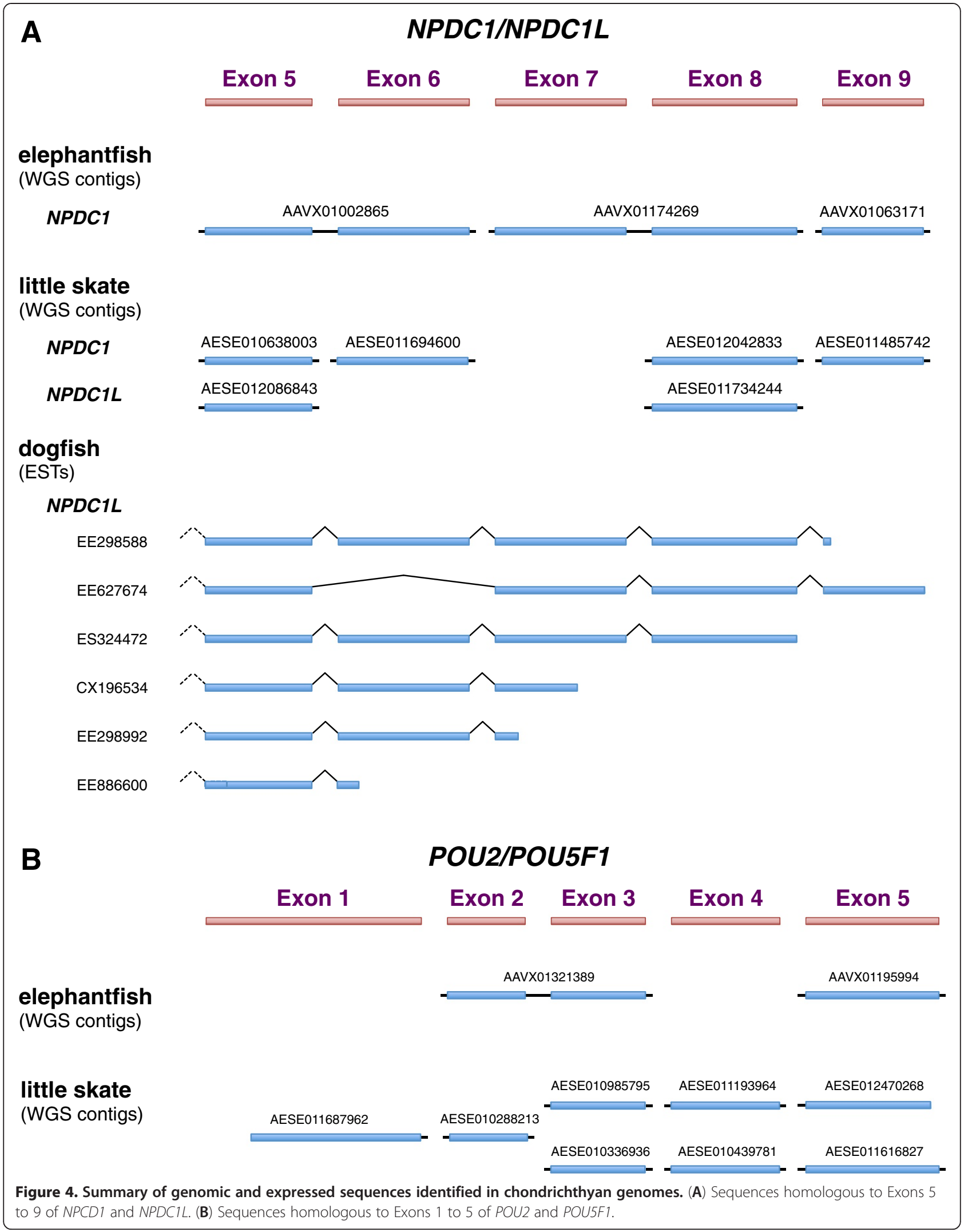




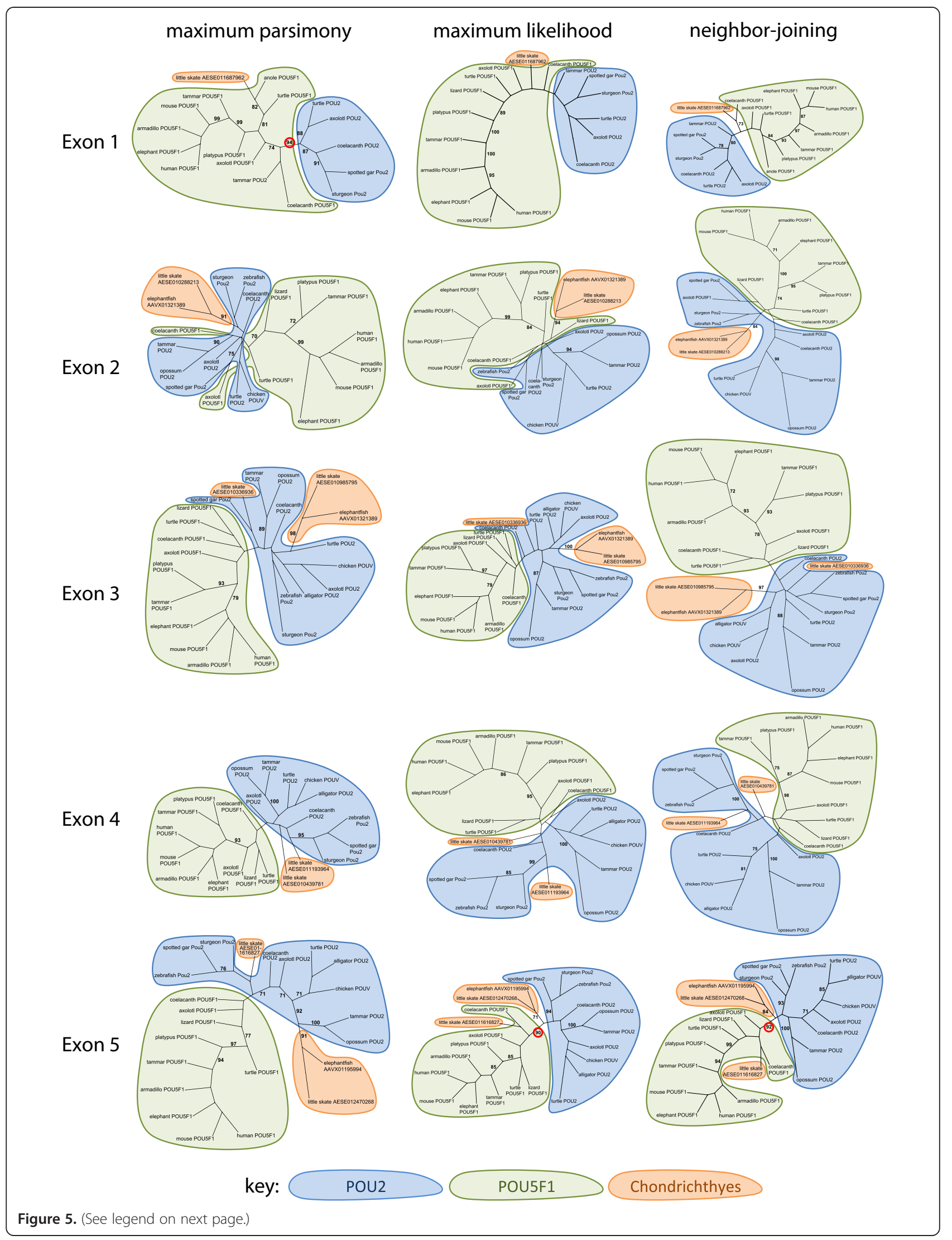


(See figure on previous page.)

Figure 5. Phylogenetic analysis of POU2/POU5F1 homologs in gnathostomes. Translated sequences of individual exons were analyzed by maximum parsimony, maximum likelihood and neighbor-joining methods and displayed as unrooted consensus trees. Alignments for the analyses are presented in Additional file 3. Only significant bootstrap values (>70\%) are shown and those relevant to the text are circled in red.

A proposed model for the evolution of the POU2/ POU5F1 family in vertebrates, based on the present data, is summarized in Figure 6. Turtles, coelacanths and probably at least some elasmobranch fishes all have orthologs of both POU2 and POU5F1, joining with marsupials, monotremes and salamanders [5-7] as the only known lineages that have retained both genes. Extinction of POU5F1 in birds and crocodilians may have been a single event dating to a common archosaurian ancestor. The absence of POU2 in both anole and python genomes also suggests a single extinction event in a common ancestor of squamate reptiles.

Contrary to a recent assertion [8], our study provides clear evidence that the pou 2 gene of teleost and other actinopterygian fishes is a bona fide ortholog of tetrapod POU2 and not of POU5F1. Its recent renaming to pou5f1 (RefSeq-ID NM_131112.1) by the zebrafish nomenclature committee is, therefore, misleading. POU5F1 became extinct possibly in a common ancestor of actinopterygians, or at least of teleost fishes. This finding

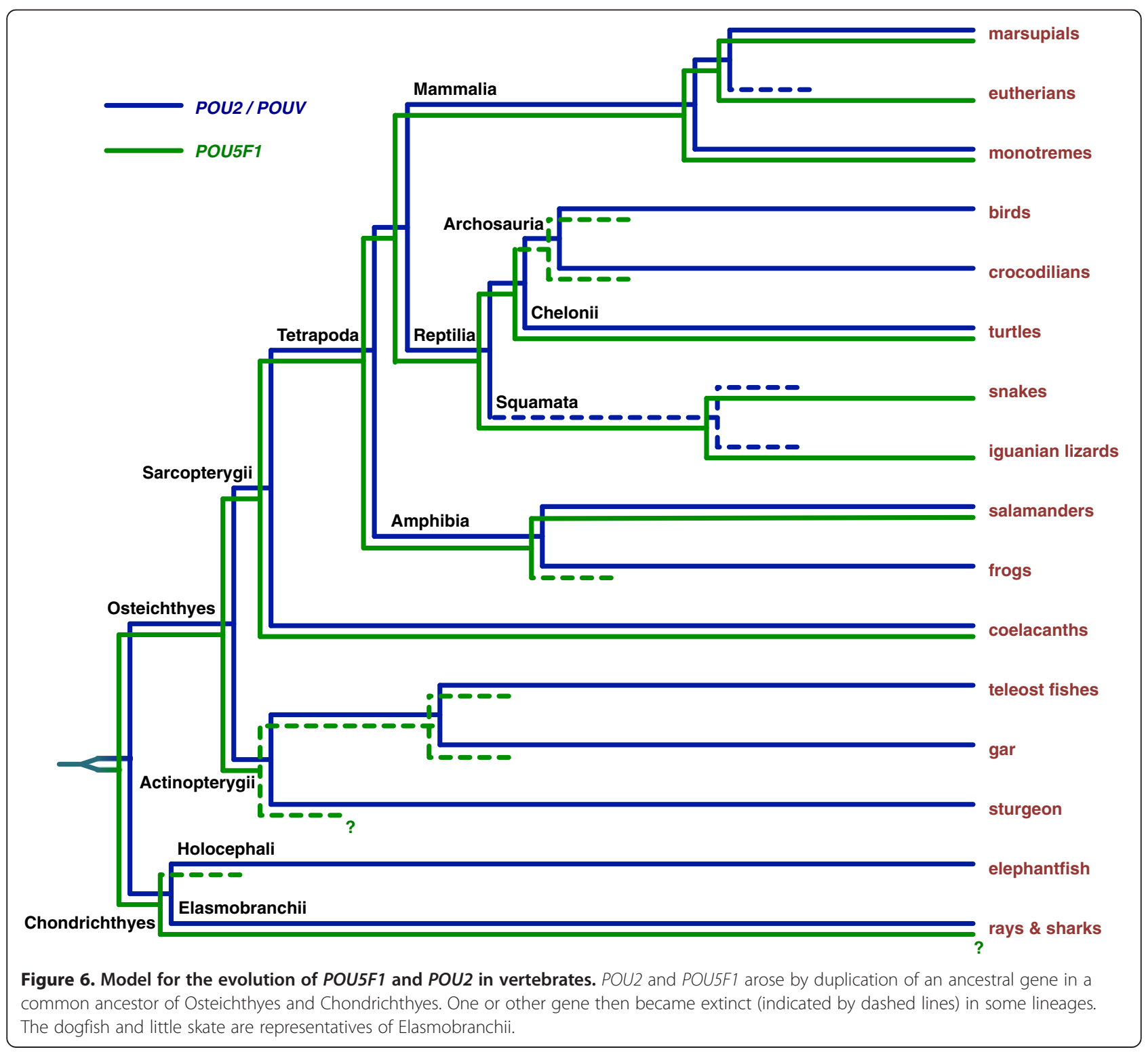


is important because misleading nomenclature can potentially lead to misleading assumptions regarding evolutionary conservation versus divergence of the respective roles of POU2 and POU5F1.

Orthologs of POU2 and POU5F1 from various vertebrates have been tested for their ability to maintain pluripotency in mouse ES cells or to generate mouse or human iPSCs. Non-eutherian POU5F1 orthologs from axolotl [7] and platypus [6] both have this ability. POU2 orthologs from opossum, chicken, Xenopus, axolotl and medaka are also able to maintain or induce pluripotency $[6,7,12,16,17]$, even in species that have retained both paralogs (axolotl and opossum). Surprisingly, although medaka pou 2 can maintain mESC pluripotency, pou 2 of another teleost fish, zebrafish, cannot [7]. Neverthess, the conservation in function of class V POU family members despite very poor sequence conservation in the transactivation domains can perhaps be expected considering that deletion of either (but not both) of the $\mathrm{N}$ - or C-terminal domains did not affect the ability of mouse POU5F1 to maintain ES cell pluripotency [18]. Maintaining pluripotency in ES cells probably serves as a model for only a limited proportion of the roles POU2 and POU5F1 serve in vivo. Thus, although there is strong evidence for an ancient role for the common ancestor of POU2 and POU5F1 at least in the maintenance of pluripotency, deducing distinct functions and roles between various $P O U 2$ and POU5F1 orthologs will probably require in vivo assays other than ES cell complementation. This would include deducing the function of the conserved (K/R)XWYXF motif in the N-terminal domain.

A general, although not universal pattern, appears to be that POU2 orthologs are more widely expressed in non-germline and non-pluripotent tissues than are POU5F1 orthologs. In marsupials, POU2 transcripts are detectable by RT-PCR in a wide range of adult tissues whereas POU5F1 expression is restricted to the germ line and early conceptuses [5,19]. Nevertheless, POU2 is also differentially expressed in early tammar conceptuses [5] and protein immunolocalization suggests that POU2 is a more specific marker than POU5F1 of very early epiblast [20]. Interestingly in the sturgeon, pou2 transcripts were also detected in many adult tissues [13].

POU2 orthologs seem to have a more important role than POU5F1 in early neural development. In the axolotl, POU2 but not POU5F1 is expressed specifically in the early neural plate and later in the developing hindbrain [7], in a pattern similar to chicken POUV [12], Xenopus OCT25 and OCT91 [17] and zebrafish pou 2 [21-25], but not medaka pou2 [26].

The pattern of germ cell expression is also inconsistent among POU2 and POU5F1 orthologs. Marsupial POU5F1 but not POU2 expression was detected by in situ hybridization in primordial germ-cells and early spermatogonia $[5,19]$, whereas both axolotl paralogs are expressed in germ cells [7]. Germ cell expression has also been reported for chicken POUV (POU2) and Xenopus OCT60 [27] and among teleosts for medaka [26] and cod [28] but not for zebrafish. Nevertheless, all POU5F1 orthologs that have been examined are expressed in germ cells, which may be significant. Two modes of germ cell specification are recognized among vertebrates - predetermined (germ plasm) and inductive (regulative). In the predetermined mode, maternally inherited germ plasm is partitioned during cleavage to a subset of cells, which are then specified to become germ cells. In the inductive mode, there is no germ plasm and germ cells become specified by inducing signals from neighboring cells. The inductive mode is considered ancestral, with the predetermined mode independently derived in birds, frogs and teleost fishes [29]. The predetermined mode was proposed to be correlated with a derived mode of mesoderm induction [30,31] as well as with a more POU5F1-like class V POU transcription factor [32], although this preceded knowledge of the paralogous relationship between POU2 and POU5F1 among vertebrates $[5,6]$. We thus hypothesized that inductive germ cell specification is specifically correlated with the presence of a POU5F1 ortholog, irrespective of the presence of POU2 [5]. Our present data are still largely consistent with this hypothesis. Evidence suggests that turtles have inductive germ cell specification [33], while retaining POU5F1 (and POU2). To our knowledge, no data exist on the mode of germ cell specification in crocodilians, which would be expected to share a similar mode with birds. Evidence suggests that the sturgeon (a basal actinopterygian) lacks germ plasm and is thus likely to have the inductive mode of germ cell specification $[31,34]$. The sturgeon genome has not been sequenced, so it is possible that it has a POU5F1 ortholog in addition to its previously reported POU2 ortholog [13]. Indeed, Johnson et al. [32] do refer to an unpublished "Oct-4" sequence from sturgeon. In the sequenced genome of the spotted gar (a less basal, non-teleost actinopterygian), we found all five exons of a POU2 ortholog but no exons corresponding to a POU5F1 ortholog. Thus POU2 presumably became extinct in a common ancestor of gars and teleost fishes. To our knowledge, the mode of germ cell specification of gars has not been investigated. Early studies of elasmobranch fishes cited by Extavour and Akam [29] drew conflicting conclusions regarding the mode of germ cell specification in elasmobranch fishes and no studies have examined fishes of the subclass Holocephali (for example, elephantfish). Further studies examining the mode of germ cell specification in several of the above lineages will provide powerful data to test the intriguing notion that the acquisition of predetermined germ cell specification permits or even drives the loss of POU5F1 [32]. 


\section{Conclusions}

Our study resolves the controversial evolutionary relationship between teleost pou2 and tetrapod POU2 and POU5F1. It shows that class V POU transcription factors have existed at least since the common ancestor of gnathostome vertebrates and provides a framework for elucidating the basis for the lineage-specific extinctions of POU2 and POU5F1, which is likely to be informative for understanding their roles in development.

\section{Methods}

General sequence analysis was performed using MacVector software, version 12.7.3 (MacVector, Inc.; Cary, North Carolina, USA). Sources of all sequences are detailed in Additional file 4. Sequences were selected to provide a broad range of taxonomic groups. The three Xenopus POU2 orthologs (OCT91, OCT60 and OCT25) were not included in analyses since they display considerable sequence divergence, which could be related to redundancy among them. All alignments were performed using the algorithm Muscle (with default parameters) in MacVector on translated sequences. Subsequent manual adjustment was only performed for the full POU2/POU5F1 alignment. Phylogenetic analyses on aligned sequences were performed using PHYLIP version 3.69 [35], using the maximum parsimony (100 replicates, 10 jumbles), maximum likelihood without the assumption of a molecular clock (1,000 replicates, 10 jumbles) and neighbor-joining (1,000 replicates) methods with default parameters.

\section{Additional files}

Additional file 1: Full alignment of class V POU family translated sequences. Within the N-terminal domain, the only motif present in all family members is boxed (A). Within the POU domain, position (B) is a glutamic acid residue in all POU2 orthologs and an aspartic acid residue in all POU5F1 orthologs. Single-residue deletions at (C) and (D) are specific to boroeutherian POU5F1 and all POU5F1 orthologs, respectively. Additional file 2: NPDC1 and NPDC1L alignments. Alignments of NPDC1 and NPDC1L sequences used for phylogenetic analyses.

Additional file 3: POU2 and POU5F1 alignments. Alignments of class $\checkmark$ POU family translated sequences used for phylogenetic analyses.

Additional file 4: Sequence sources. Sources of all sequences used in this study.

\section{Abbreviations}

ES cells: Embryonic stem cells; ESTs: Expressed sequence tags; IPSCs: induced pluripotent stemcells; WGS: Whole genome shotgun.

\section{Competing interests}

The authors declare that they have no competing interests.

\section{Authors' contributions}

SF conceived the study, performed all analyses and drafted the manuscript. SF and MBR contributed to interpretation of the data and the writing of the manuscript. Both authors read and approved the final manuscript.
Acknowledgements

We thank Dr. Natalia Tapia for providing the sequence of axolotl POU2.

Published: 9 May 2013

\section{References}

1. Nichols J, Zevnik B, Anastassiadis K, Niwa H, Klewe-Nebenius D, Chambers I, Scholer H, Smith A: Formation of pluripotent stem cells in the mammalian embryo depends on the POU transcription factor Oct4. Cell 1998, 95:379-391.

2. Takahashi K, Tanabe K, Ohnuki M, Narita M, Ichisaka T, Tomoda K, Yamanaka S: Induction of pluripotent stem cells from adult human fibroblasts by defined factors. Cell 2007, 131:861-872.

3. Yu J, Vodyanik MA, Smuga-Otto K, Antosiewicz-Bourget J, Frane JL, Tian S, Nie J, Jonsdottir GA, Ruotti V, Stewart R, Slukvin II, Thomson JA: Induced pluripotent stem cell lines derived from human somatic cells. Science 2007, 318:1917-1920.

4. Kehler J, Tolkunova E, Koschorz B, Pesce M, Gentile L, Boiani M, Lomelí H, Nagy A, McLaughlin KJ, Schøler HR, Tomilin A: Oct4 is required for primordial germ cell survival. EMBO Rep 2004, 5:1078-1083.

5. Frankenberg S, Pask A, Renfree MB: The evolution of class V POU domain transcription factors in vertebrates and their characterisation in a marsupial. Dev Biol 2010, 337:162-170.

6. Niwa H, Sekita Y, Tsend-Ayush E, Grutzner F: Platypus Pou5f1 reveals the first steps in the evolution of trophectoderm differentiation and pluripotency in mammals. Evol Dev 2008, 10:671-682.

7. Tapia N, Reinhardt P, Duemmler A, Wu G, Araúzo-Bravo MJ, Esch D, Greber B, Cojocaru V, Rascon CA, Tazaki A, Kump K, Voss R, Tanaka EM, Schöler HR: Reprogramming to pluripotency is an ancient trait of vertebrate Oct4 and Pou2 proteins. Nat Commun 2012, 3:1279.

8. Onichtchouk D: Pou5f1/oct4 in pluripotency control: insights from zebrafish. Genesis 2012, 50:75-85.

9. Okamoto K, Okazawa H, Okuda A, Sakai M, Muramatsu M, Hamada H: A novel octamer binding transcription factor is differentially expressed in mouse embryonic cells. Cell 1990, 60:461-472.

10. Okazawa H, Okamoto K, Ishino F, Ishino-Kaneko T, Takeda S, Toyoda Y, Muramatsu M, Hamada $\mathrm{H}$ : The oct3 gene, a gene for an embryonic transcription factor, is controlled by a retinoic acid repressible enhancer. EMBO J 1991, 10:2997-3005.

11. Scholer HR, Ruppert S, Suzuki N, Chowdhury K, Gruss P: New type of POU domain in germ line-specific protein Oct-4. Nature 1990, 344:435-439.

12. Lavial F, Acloque H, Bertocchini F, Macleod DJ, Boast S, Bachelard E, Montillet G, Thenot S, Sang HM, Stern CD, Samarut J, Pain B: The Oct4 homologue PouV and Nanog regulate pluripotency in chicken embryonic stem cells. Development 2007, 134:3549-3563.

13. Ye H, Du H, Chen XH, Cao H, Liu T, Li CJ: Identification of a pou2 ortholog in Chinese sturgeon, Acipenser sinensis and its expression patterns in tissues, immature individuals and during embryogenesis. Fish Physiol Biochem 2012, 38:929-942.

14. Dupont E, Sansal I, Evrard C, Rouget P: Developmental pattern of expression of NPDC-1 and its interaction with E2F-1 suggest a role in the control of proliferation and differentiation of neural cells. J Neurosci Res 1998, 51:257-267.

15. Galiana E, Vernier $P$, Dupont $E$, Evrard C, Rouget P: Identification of a neuralspecific cDNA, NPDC-1, able to down-regulate cell proliferation and to suppress transformation. Proc Nat Acad Sci U S A 1995, 92:1560-1564.

16. Hammachi F, Morrison GM, Sharov AA, Livigni A, Narayan S, Papapetrou EP, O'Malley J, Kaji K, Ko MS, Ptashne M, Brickman JM: Transcriptional activation by Oct4 is sufficient for the maintenance and induction of pluripotency. Cell Rep 2012, 1:99-109.

17. Morrison GM, Brickman JM: Conserved roles for Oct4 homologues in maintaining multipotency during early vertebrate development. Development 2006, 133:2011-2022.

18. Niwa H, Masui S, Chambers I, Smith AG, Miyazaki J: Phenotypic complementation establishes requirements for specific POU domain and generic transactivation function of Oct-3/4 in embryonic stem cells. Mol Cell Biol 2002, 22:1526-1536.

19. Frankenberg $S$, Tisdall D, Selwood L: Identification of a homologue of POU5F1 (OCT3/4) in a marsupial, the brushtail possum. Mol Reprod Dev 2001, 58:255-261. 
20. Frankenberg S, Shaw G, Freyer C, Pask AJ, Renfree MB: Early cell lineage specification in a marsupial: a case for diverse mechanisms among mammals. Development 2013, 140:965-975.

21. Burgess S, Reim G, Chen W, Hopkins N, Brand M: The zebrafish spiel-ohne-grenzen (spg) gene encodes the POU domain protein Pou2 related to mammalian Oct4 and is essential for formation of the midbrain and hindbrain, and for pre-gastrula morphogenesis. Development 2002, 129:905-916.

22. Hauptmann G, Belting HG, Wolke U, Lunde K, Soll I, Abdelilah-Seyfried S, Prince V, Driever W: Spiel ohne grenzen/pou2 is required for zebrafish hindbrain segmentation. Development 2002, 129:1645-1655.

23. Hauptmann G, Gerster T: Pou-2-a zebrafish gene active during cleavage stages and in the early hindbrain. Mech Dev 1995, 51:127-138

24. Reim G, Brand M: Spiel-ohne-grenzen/pou2 mediates regional competence to respond to Fgf8 during zebrafish early neural development. Development 2002, 129:917-933.

25. Takeda H, Matsuzaki T, Oki T, Miyagawa T, Amanuma H: A novel POU domain gene, zebrafish pouz: expression and roles of two alternatively spliced twin products in early development. Genes Dev 1994, 8:45-59.

26. Sanchez-Sanchez AV, Camp E, Garcia-Espana A, Leal-Tassias A, Mullor JL: Medaka Oct4 is expressed during early embryo development, and in primordial germ cells and adult gonads. Dev Dyn 2010, 239:672-679.

27. Hinkley CS, Martin JF, Leibham D, Perry M: Sequential expression of multiple POU proteins during amphibian early development. Mol Cell Biol 1992, 12:638-649.

28. Holen E, Kausland A, Skjaerven K: Embryonic stem cells isolated from Atlantic cod (Gadus morhua) and the developmental expression of a stage-specific transcription factor ac-Pou2. Fish Physiol Biochem 2010, 36:1029-1039.

29. Extavour CG, Akam M: Mechanisms of germ cell specification across the metazoans: epigenesis and preformation. Development 2003, 130:5869-5884.

30. Johnson AD, Drum M, Bachvarova RF, Masi T, White ME, Crother BI: Evolution of predetermined germ cells in vertebrate embryos: implications for macroevolution. Evol Dev 2003, 5:414-431.

31. Johnson AD, Richardson E, Bachvarova RF, Crother BI: Evolution of the germ line-soma relationship in vertebrate embryos. Reproduction 2011 141:291-300.

32. Johnson AD, Crother B, White ME, Patient R, Bachvarova RF, Drum M, Masi $T$ : Regulative germ cell specification in axolotl embryos: a primitive trait conserved in the mammalian lineage. Philos Trans R Soc Lond 2003, 358:1371-1379.

33. Bachvarova RF, Crother BI, Manova K, Chatfield J, Shoemaker CM, Crews DP, Johnson AD: Expression of Dazl and Vasa in turtle embryos and ovaries: evidence for inductive specification of germ cells. Evol Dev 2009, 11:525-534.

34. Zelazowska M, Kilarski W, Bilinski SM, Podder DD, Kloc M: Balbiani cytoplasm in oocytes of a primitive fish, the sturgeon Acipenser gueldenstaedtii, and its potential homology to the Balbiani body (mitochondrial cloud) of Xenopus laevis oocytes. Cell Tissue Res 2007, 329:137-145.

35. PHYLIP (Phylogeny Inference Package) version 3.6. [http://www.phylip.com/].

doi:10.1186/1741-7007-11-56

Cite this article as: Frankenberg $S$ and Renfree MB: On the origin of POU5F1. BMC Biology 2013 11:56. 